\section{Promotion de la santé et diagnostic précoce du cancer chez les aînés: une approche pratique}

\author{
par Margaret Muir, Marlene Greenberg, Susan Plante, \\ Margaret Fitch, Linda Levstein et Eva King
}

\section{Abrégé}

On sait peu de choses au sujet des connaissances, attitudes et comportements sanitaires des aînés (personnes de plus de 55 ans) en matière de cancer et au sujet de l'élaboration d'interventions répondant à leurs besoins en éducation. On a mis sur pied un projet collectif dont le but était de concevoir un programme pertinent pour les personnes âgées. Les travaux initiaux comprenaient une évaluation des besoins de la collectivité par le biais de groupes de réflexion, d'entrevues individuelles et d'instruments d'auto-évaluation. On a fait appel à des intervenants communautaires clés entretenant des rapports avec des organismes afin de trouver des sujets pour l'étude. Cette dernière inclut divers groupes ethnoculturels, des groupes de personnes à faible revenu, des personnes isolées socialement ainsi que d'autres groupes pré-établis. L'évaluation des besoins a révélé ce qui suit: les gens ne voient pas dans l'âge un facteur de risque de cancer; le transport fait obstacle au dépistage; le dépistage fait peur aux gens; les médecins représentent la source principale de connaissances spécialisées sur le cancer et ils contrólent l'accès au dépistage; la famille et les pairs sont les principales sources de soutien; les groupes ethniques ont des besoins différents en matière d'information; et enfin, les suggestions visant à améliorer le mode de vie réduisent le risque de cancer. Les résultats des travaux indiquent que les programmes communautaires de promotion de la santé pour les aînés doivent employer des approches multiples et combiner des stratégies $s$ 'ils veulent répondre à leurs besoins en éducation.

\section{Introduction}

L'âge est un important facteur de risque de cancer. Par conséquent, le cancer affectera un nombre croissant de Canadiens et Canadiennes étant donné le vieillissement constant de la population du pays. II importe donc d'instruire les personnes à risque au sujet de la maladie. Pourtant, on sait actuellement peu de choses à propos des connaissances, attitudes et comportements sanitaires des personnes âgées en matière de cancer et à propos de la conception d'interventions de promotion de la santé et de diagnostic précoce qui répondent à leurs besoins particuliers (Comité d'experts de Cancer 2000, 1992; Dellefield, 1988; Mettlin, 1991; Rimer, Jones, Wilson et al, 1991). Récemment, le Comité d'experts de Cancer 2000 (1992) a déclaré sur le cancer chez les défavorisés qu'il était urgent d'examiner les obstacles à la promotion de la santé, à la réduction du risque de cancer et au diagnostic précoce chez les aînés et de mettre sur pied des programmes répondant à leurs besoins. Dellefield (1988) mentionne les obstacles à la réduction du risque de cancer et au diagnostic précoce chez les aînés. Ces obstacles incluent certains comportements sanitaires associés aux personnes âgées, les pratiques d'une partie des professionnels de la santé et les limitations inhérentes au système de soins de santé. De nombreux aînés ne se rendent pas compte qu'ils courent un risque accru d'avoir le cancer et négligent ainsi de participer aux programmes de diagnostic et d'effectuer des auto-examens (Williams \& Dueker, 1985; Morra \& Blumberg, 1991). Les consommateurs de soins et les professionnels de la santé voient souvent dans les changements des signes de vieillissement alors que ceux-ci sont de nature pathologique (Satariano, Belle \& Swanson, 1986). Les infirmières et les médecins peuvent ignorer que l'âge s'accompagne d'un risque substantiel d'être atteint du cancer et peuvent penser que dans ces cas-là, la maladie n'exige pas une intervention agressive.

En général, les programmes de promotion de la santé et de diagnostic visent les groupes moins âgés, mais, de nos jours, les aînés réalisent que les changements qu'ils éprouvent sont peut-être dus à l'absence de pratiques de promotion de la santé dans le passé. D'autres facteurs influent sur les comportements en matière de diagnostic; on peut les relier aux variations que connaît cette population dans les domaines du revenu, du logement, de l'éducation, de la capacité physique, de la diversité des centres d'intérêt et des antécédents ethnoculturels (Gray, 1985; Dellefield, 1988). Il existe, jusqu'à présent, peu d'écrits sur la prévention et le diagnostic précoce du cancer chez les personnes âgées. La planification des programmes de promotion de la santé et de lutte contre le cancer à l'intention des aînés doit tenir compte de toutes ces questions, notamment la problématique homme-femme et les questions socioéconomiques et ethnoculturelles (Given \& Given, 1989; White, Fishman, Guthrie \& Fagan, 1993). Cet article décrit la méthode retenue en milieu urbain pour planifier un programme de promotion de la santé et de lutte contre le cancer à l'intention des personnes âgées. Les étapes suivies et les leçons tirées de cette expérience pourront aider des infirmières à mettre en oeuvre un programme similaire.

Quoiqu'on reconnaisse que le facteur de risque s'accroît chez les personnes âgées de plus de 65 ans, on estime que la promotion de la santé utilisée à titre de stratégie d'intervention doit s'appliquer aux personnes de la décennie précédente. On a donc sélectionné pour ce projet des personnes ayant plus de 55 ans.

Pour qu'un programme communautaire réussisse, il faut que la collectivité soit impliquée dès la phase d'élaboration. C'est pourquoi nous avons favorisé la participation de représentants communautaires du début à la fin du projet.

Notre projet avait les objectifs suivants:

- établir un groupe de travail sur l'évaluation des besoins qui soit axé sur la collectivité

- examiner les obstacles à la promotion de la santé et au diagnostic précoce du cancer chez les adultes au sein d'une population hétérogène - engager la collectivité dans la détermination des besoins et des solutions en matière de promotion de la santé

- accéder aux ressources communautaires existantes et collaborer avec elles

- former un groupe consultatif du programme chargé de dégager les stratégies permettant de répondre aux besoins définis dans le cadre de l'évaluation des besoins

- évaluer l'efficacité des activités du projet.

Il est essentiel de bien cerner la collectivité visée et ses besoins dès le départ si on veut que la planification du programme soit réussie.

Margaret Muir, RN, MHSc, oeuvre au Health Information Centre, Sunnybrook Health Science Centre (elle était à la Anne Johnson Health Station auparavant); Marlene Greenberg, MS, et Margaret Fitch, RN, PhD, travaillent également au Toronto-Sunnybrook

Health Science Centre; Susan Plante, RN, MSN, oeuvre au Service de santé publique de North York;

Linda Levstein était l'animatrice communautaire; et enfin, Eva King représentait la collectivité. 


\section{Processus}

Le projet comprenait les étapes suivantes (voir la figure 1):

\section{Le groupe de travail sur l'évaluation des besoins}

Le groupe de travail regroupait des représentants du Centre régional de cancérologie de Toronto-Sunnybrook, du North York Public Health Department, de la Anne Johnson Health Station (un centre de soins communautaire) et une personne âgée provenant d'un organisme communautaire local. Le rôle principal du groupe de travail consistait à encadrer l'évaluation des besoins de la collectivité. Cette évaluation avait les trois objectifs suivants:

1) rassembler des données sur les connaissances, attitudes et comportements sanitaires des aînés en matière de promotion de la santé et de diagnostic précoce du cancer

2) découvrir la ou les meilleure(s) méthode(s) permettant de dégager les idées et opinions des aînés

3) déterminer les stratégies qui aideront les aînés à apprendre une information nouvelle dans le cadre d'un programme.

Le groupe de travail sur l'évaluation des besoins a embauché une animatrice chargée de réaliser l'évaluation des besoins. Celle-ci a su établir et maintenir des contacts en gagnant la confiance des résidents et des prestataires de services locaux. L'animatrice a également consigné les données de l'évaluation des besoins et a évalué en permanence le processus d'évaluation (en collaboration avec le groupe de travail).

Figure 1: Schéma des activités du projet

\begin{tabular}{|l|}
\multicolumn{1}{|c|}{ Formation du groupe de } \\
travail sur l'évaluation des besoins \\
- a défini ses buts, objectifs et mandat \\
- a précisé le type d'information à recueillir \\
- a contribué à l'évaluation des besoins \\
\hline
\end{tabular}

\section{Groupe de réflexion d'intervenants}

- a validé les idées et décisions initiales auprès des membres de la population concernée

- a déterminé les types de questions à poser

- s'est penché sur l'emploi du format "groupe de réflexion" avec des personnes âgées

Définition du rôle de l'animatrice communautaire

- élaboration de la description de tâche

- embauche de l'animatrice communautaire

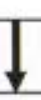

Conduite de l'évaluation de la collectivité

- profil: découverte des groupes communautaires

- réseaux: établissements de contacts au sein de la collectivité

- évaluation des besoins (groupe de réflexion, entrevues, sondages)

Formation du groupe consultatif du programme - a analysé les données d'évaluation des besoins

- a recommandé que l'élaboration du programme de promotion de la santé s'articule autour de la prévention et du diagnostic précoce du cancer chez les aînés

\section{L'évaluation de la collectivité}

Afin de valider les idées initiales du projet et d'en étendre la portée, de définir la population visée et de déterminer les moyens de la rejoindre, le groupe de travail a mis sur pied un groupe de réflexion (ou groupe d'intervenants) se composant de six aînés qui a tenu une seule et unique réunion. Cette dernière a révélé l'aisance avec laquelle les participants parlent du cancer, l'importance d'une approche collective et l'intérêt que les aînés portent à la santé.

Étant donnée la très grande taille de la zone de recrutement, on a sélectionné quatre petites communautés qui faisaient partie de la région couverte pour réaliser l'évaluation des besoins de la collectivité. Ces communautés comptaient toutes une forte concentration d'aînés d'une grande diversité en termes de revenu, d'antécédents culturels et de rapports sociaux. On a tout d'abord profité des réseaux constitués par les membres du groupe de travail pour établir les premiers contacts avec les groupes visés. Ces réseaux comprenaient des immeubles résidentiels et des centres pour personnes âgées, des organismes de santé publique et ethnoculturels, des centres de soins communautaires et des leaders communautaires informels.

Des intervenants clés, des prestataires de services, des résidents locaux et des rapports déjà publiés ont fourni l'information qui a servi à caractériser chaque communauté et ses membres. Cette information incluait la structure physique de la communauté, la condition socioéconomique, la structure familiale, les obstacles rencontrés pour obtenir des soins et les services de santé dont disposent les aînés.

\section{Une évaluation des besoins faisant appel à plusieurs méthodes}

On a utilisé trois méthodes pour mener à bien l'évaluation des besoins de la collectivité: des groupes de réflexion, des entrevues et un sondage par auto-évaluation. Elles feront chacune l'objet d'une brève description. II convient de consulter Fitch, Greenberg, Levstein et al (1996) pour connaître les résultats obtenus à l'aide de chacune de ces méthodes.

\section{Les groupes de réflexion}

On a tenu dix groupes de réflexion auxquels ont assisté 158 aînés. Six de ces groupes étaient composés de groupes d'aînés qui se réunissent régulièrement en tant que tels. La seule raison d'être des groupes de réflexion était de connaître l'opinion des aînés sur différents sujets. Les quatre derniers groupes ont été formés exprès pour le projet et organisés dans le but de rejoindre d'autres groupes culturels, car on a découvert que la culture influençait les idées des personnes âgées en matière de santé. On a recruté ces groupes culturels, qui se sont révélés d'un accès plus difficile, dans les communautés italienne, grecque, hispanique et chinoise. Les groupes de réflexion tenaient une séance d'environ une heure et demie sauf dans le cas des groupes culturels où on faisait appel à des interprètes. Dans tous les cas, l'animation des groupes était assurée, en partie, par une personne âgée.

Durant ces séances, les groupes de réflexion se sont penchés sur les thèmes suivants: connaissances, croyances, attitudes, comportements et stratégies des aînés en matière de prévention et de diagnostic précoce du cancer et obstacles rencontrés. On y abordait aussi les frottis vaginaux, la mammographie, les cancers du testicule, de la prostate, de la peau, de l'intestin et du côlon. Les discussions enrichissantes permettaient d'examiner en profondeur les questions à l'étude.

\section{Les entrevues}

On a interviewé neuf personnes en tout dont trois connaissent un certain isolement social et les six autres mènent une vie active. Ces entrevues, qui duraient à peu près une heure, ont été réalisées au domicile des répondants. On a fait appel à des interprètes dans deux cas.

On a effectué les entrevues individuelles pour voir s'il y avait des différences au niveau de la quantité et du type d'information recueillie par rapport aux résultats des groupes de réflexion. On a également profité de ce format pour rejoindre les aînés isolés socialement. On a 
choisi les interviewés de manière à représenter l'éventail de statuts économiques et d'antécédents culturels de la collectivité prise dans son ensemble. On a obtenu le nom de ces personnes auprès d'organismes communautaires. Lors des entrevues, on posait les mêmes questions que dans le cadre des groupes de réflexion.

\section{Le sondage d'auto-évaluation}

L'évaluation des connaissances des aînés relatives au cancer et au dépistage du cancer exigeait un instrument facile à administrer. La stratégie retenue pour le sondage était celle du papier et crayon. Le groupe de travail a recommandé de rejoindre un grand échantillon de la population visée, de voir s'il était possible d'utiliser cette méthode pour recueillir des données auprès de personnes âgées et de voir s'il existait des différences au niveau des connaissances et pratiques personnelles entre les données ainsi recueillies et celles obtenues dans le cadre des groupes de réflexion et des entrevues. On a recensé les écrits afin de trouver un instrument à la fois court et facileà utiliser. Le groupe de travail a recommandé l'utilisation du sondage mis au point par Kane-Williams et White (1983) parce que ce demier portait sur des sujets semblables à ceux qui sous-tendaient les groupes de réflexion et les entrevues. On s'est servi des critères suivants pour sélectionner le sondage qui avait déjà été utilisé avec des aînés: s'intéresse à plus d'un type et à plus d'un siège de cancer; est simple et bref; comprend des questions "vrai-faux" et/ou à choix multiples; est facile à administrer; se penche sur les connaissances et les pratiques en matière de cancer; et enfin, est d'une fidélité ct d'une validité prouvées.

On y a ajouté des questions de nature démographique avant de le faire traduire dans les langues représentatives de la collectivité (italien, grec, panjabi, hébreu, russe, chinois et espagnol). Pour nous assurer de la qualité de la traduction, nous avons demandé à une autre personne de retraduire le texte vers l'anglais. L'animatrice communautaire s'est chargée de faire distribuer le sondage par l'intermédiaire d'agents communautaires. Il s'agissait là d'une méthode efficace et rentable. Chaque formulaire de sondage incluait un petit cadeau incitatif. Nos efforts se sont soldés par d'excellents résultats: ainsi, 588 formulaires nous ont été renvoyés sur les 783 distribués.

\section{Leçons tirées de l'expérience}

Nous avons pu tirer plusieurs leçons de la première partie du projet. Premièrement, les domaines d'incertitude dégagés en matière de prévention et de diagnostic précoce du cancer étaient les mêmes dans les groupes de réflexion, les entrevues et le sondage. Les groupes de réflexion se sont traduits par des échanges enrichissants et un grand soutien qu'on ne retrouvait pas dans le contexte des entrevues individuelles. Toutefois, ces entrevues ont donné lieu à des échanges plus personnels. Les répondants ont trouvé que le formulaire était facile à remplir. La traduction du formulaire en plusieurs langues s'est révélée être un atout. Le sondage pourrait servir d'instrument de mesure des connaissances relatives au cancer dans d'autres études et dans d'autres collectivités. L'emploi de plusieurs méthodes de collecte de données a fourni une masse importante de données qui n'a fait que rehausser l'intégralité des données, la complexité de l'analyse et la richesse des résultats.

Deuxièmement, les résultats indiquent que les connaissances des aînés relatives au cancer et à sa prévention comportent des lacunes et des idées fausses, mais que beaucoup d'entre eux ont hâte de s'instruire. Bien que l'âge figure parmi les facteurs de risque de cancer, la grande majorité des participants ne le considéraient pas comme tel. Ils étaient nombreux àvouloir se tourner vers leur médecin pour obtenir des réponses aux questions qu'ils se posent sur le cancer alors que d'autres estimaient que leur médecin ne leur parlait pas de dépistage au cours de leurs visites médicales régulières. Un grand nombre d'entre eux pensaient que les gens évitent le dépistage par peur, ils se tourneraient vers leur entourage (famille, amis) pour se rassurer. Les hommes hésitaient plus que les femmes à parler du cancer et à admettre leur peur face à la maladie. Les membres des groupes ethnoculturels ont souligné qu'ils avaient des besoins différents en matière d'information. Ils ont également réclamé des séances et du matériel pédagogiques dans leur propre langue tout en suggérant l'utilisation d'une information visuelle.

Troisièmement, la communauté des aînés nous a fourni une aide inestimable quant à la conduite même de la collecte des données. Voici quelques-unes des idées mentionnées:
1) les réunions de groupe sont des événements sociaux et les rafraichissements en sont un aspect important;

2) une considération importante est de tenir les groupes de réflexion là où se trouvent les aînés, plutôt que de leur demander de se rendre à l'extérieur;

3 ) inclure un petit cadeau pour encourager les gens à remplir le formulaire de sondage est un geste amical;

4) les invitations personnelles à assister aux sessions collectives sont plus efficaces que les affiches et les dépliants;

5) l'opinion des aînés isolés socialement compte et doit être reflétée dans les données.

Quatrièmement, le travail en commun avec plusieurs organismes a rehaussé la productivité du projet. Les membres de chaque organisme ont apporté des compétences et connaissances variées. Aussi, les organismes se sont partagés les tâches en vue de réaliser le projet (photocopie, courrier, secrétariat, lieux de réunion, analyse des données, etc.) et aucun d'entre eux n'a eu à assumer la responsabilité totale du projet.

Enfin, il faut beaucoup de temps pour rejoindre les groupes de personnes à faible revenu et les communautés ethnoculturelles et gagner leur confiance; il est donc préférable d'utiliser les réseaux existants. En dernière analyse, cela valait la peine de consacrer tant de temps à la définition et à la compréhension des problèmes tels que vécus au sein de ces collectivités.

\section{Communication des résultats}

Une fois l'évaluation des besoins de la collectivitê terminée, on a demandé aux membres de la collectivité et des organismes communautaires de participer aux activités du groupe consultatif du programme. Ce groupe se composait d'aînés et de prestataires de services oeuvrant dans le domaine du cancer ou auprès de personnes âgées. Le groupe consultatif véhiculait les résultats de l'évaluation des besoins dans la collectivité et s'assurait de la pertinence des recommandations du groupe de travail visant l'évaluation des besoins. Après avoir étudié l'ensemble des données de l'évaluation, il a suggéré des stratégies pour rejoindre les aînés.

Voici quelques-unes de ses suggestions:

- Ies organismes communautaires pertinents collaborent entre eux pour encourager la participation et la mobilisation des aînés;

- concevoir des messages positifs, pleins d'espoir, amusants et cohérents afin de surmonter le sentiment de peur éprouvé par les aînés; - fournir des renseignements sur la façon dont ils peuvent participer au dépistage et le moment opportun de le faire afin de réduire la dépendance des aînés vis-à-vis du médecin, perçu comme l'unique voie d'accès à ce genre de service;

- aborder séparément les questions intéressant les hommes;

- les aînés doivent se sentir rassurés: il importe donc qu'ils entretiennent des rapports étroits avec des pairs;

- les clés de l'accès aux groupes ethnoculturels: du matériel dans leur langue, une information visuelle et des intervenants clés issus de ces communautés; - rejoindre les personnes âgées isolées socialement et favoriser leur participation par le biais des programmes existants (service de repas à domicile et d'aide ménagère, etc.) afin de réduire l'obstacle présenté par le transport;

- faire appel à un(e) porte-parole bien connu(e), utiliser des événements spéciaux et les médias pour faire passer les messages sur le cancer à la fois positifs et captivants.

\section{Conclusion}

Notre projet avait pour but de planifier un programme de prévention et de diagnostic précoce du cancer qui réponde aux besoins des aînés. Mais avant d'y parvenir, il fallait recueillir l'information se rapportant aux connaissances, attitudes et comportements sanitaires des personnes âgées dans le domaine du cancer et aux interventions possibles qui sauraient répondre à leurs besoins en matière de promotion de la santé et de diagnostic précoce. L'évaluation des besoins de la collectivité, le groupe consultatif du programme et l'étude des aînés démontrent clairement que des recherches supplémentaires sont nécessaires dans ce domaine, car il estévident que nous ne disposons pas de connaissances suffisantes sur la prévention et le diagnostic précoce du cancer chez les aînés.

Le recours à des intervenants communautaires clés a facilité la tenue, en temps opportun, d'une évaluation des besoins qui a permis d'approfondir l'information obtenue et d'en prolonger la portée. L'inclusion de groupes 
ethnoculturels a pris plus de temps et exigé plus d'efforts de planification pour organiser la traduction et les interprètes. Ces efforts en valaient la peine, car ils nous ont permis de parvenir à une meilleure compréhension des questions de santé au sein des groupes visés. L'inclusion de groupes à faible revenu a, elle aussi, occasionné des efforts additionnels qui se sont traduits par une meilleure appréciation des questions de santé à l'étude.

La communication permanente des résultats aux membres de la collectivité a joué un rồle important dans la validation des besoins de la communauté et de ses recommandations subséquentes. Nous avons apprécié la rétroaction du groupe de travail et du groupe consultatif représentant la population visée et les membres concernés de la collectivité et nous recommandons la mise en place d'un tel mécanisme dans les projets similaires à venir. La phase suivante de nos travaux occasionnera de nouveaux contacts avec les aînés et les organismes communautaires pertinents afin qu'ils puissent sélectionner les stratégies d'action qui conviennent le mieux à leur collectivité.

Le travail collectif entrepris comprendre la collectivité a démontré que la mise en oeuvre efficace de programmes communautaires de promotion de la santé exige l'adoption de plusieurs approches, la combinaison de stratégies et le renforcement dans des cadres communautaires différents. En particulier, les aînés doivent jouer un rôle actif pour ce qui est de la programmation, en s'appuyant sur les connaissances, compétences et ressources existantes. II convient de présenter le matériel dans la bonne humeur afin de réduire le sentiment d'angoisse et de souligner l'importance de partager ses sentiments avec ses pairs. Ces méthodes aident les aînés à changer leur mode de vie et à participer aux pratiques de dépistage.

Les infirmières occupent une position privilégiée pour se consacrer à l'éducation en matière de promotion de la santé, de prévention et du diagnostic précoce du cancer. Que leurs rapports professionnels les amènent à s'entretenir avec des patients individuels ou avec des groupes, elles sont à même de souligner que l'âge est un facteur de risque et que le diagnostic précoce leur offre une aide inestimable. Les infirmières pourraient mettre à profit les suggestions présentées dans cet article pour élaborer des programmes à l'intention des aînés. Le processus global pourrait aussi être utilisé dans d'autres cadres afin de mobiliser les activités communautaires en faveur de la prévention et du dépistage précoce du cancer.

\section{Remerciements}

Merci à Maureen Cava et à Natalia Krawetz d'avoir révisé le manuscrit. Le projet a bénéficié d'une subvention de la Direction de la promotion de la santé, Ministère de la Santé de l'Ontario.

Remarque: Eva King est décédée avant que le projet ne prenne fin et que le manuscrit ne soit rédigé.

\section{Bibliographie}

Cancer 2000 Expert Panel. (1992). Cancer and the Disadvantaged. Toronto: Canadian Cancer Society.

Dellefield, M.E. (1988). Informational Needs and Approaches for Early Cancer Detection in the Elderly. Seminars in Oncology Nursing; 4(3), 156-168.

Fitch, M., Greenberg, M., Levstein, L., Muir, M., Plante, S., King, E. (1996). Health Promotion and Early Detection of Cancer in Older Adults: Needs Assessment for Program Development. (submitted)

Fitch, M., Greenberg, M., Levstein, L., Muir, M., Plante, S., King, E. (1996). Health Promotion and Early Detection of Cancer in Older Adults: Assessing Knowledge About Cancer. (submitted)

Given, B. and Given, C.W. (1989). Cancer Nursing for the Elderly - A Target for Research. Cancer Nursing; 12(2):71-77.

Gray, J.A. (1985). Education for Health in Old Age. Prevention of Disease in the Elderly. London: Churchill Livingstone: 200-230.

Kane-Williams, E., White, J.E. (1983). Community-based cancer education for the elderly. Progress in Cancer Control IV: Research in the Cancer Centre. 113-122.

Mettlin, C., Bonfiglio, J., Berg, R.L., Nelson, G.R., Patterson, W.B., Richardson, J., Runer, B., Sorenson, A., Warnecke, R. (1991). Prevention and Detection in Older Persons. Cancer; 68; 2530-2533.

Morra, M.E., Blumberg, B.D. (1991). Women's Perceptions of Early Detection in Breast Cancer: How Are We Doin? Seminars in Oncoloy Nursing; 7(3):157-160.

Satariano, W.A., Belle, S.H., Swanson, G.M. (1986). The Severity of Breast Cancer at Diagnosis: A Comparison of Age and Extent of Disease in Black and White Women. American Journal of Public Health; 76(7):779-782.

Rimer, B., Jones, W.L., Wilson, C., et al. (1983). Cancer in the elderly: A cancer control challenge, in Mettlin, C., Murphy, G.P. Eds., Progress in Cancer Control IV: Research in the Cancer Centre. New York: Alan R. Liss.

White, J.E., Fishman, N.W., Guthrie, B., Fagan, L.K. (1993). Increasing Cervical Cancer Screening Among Minority Elderly. Journal of Gerontological Nursing. May: 28-34.

Williams, G.O., Dueker, D.L. (1985). The Non-Use of Free Health Screening by Rural Elderly. American Journal of Preventive Medicine; 1(4):52-57.

\section{Draft recommendations formulated at the National Prostate Cancer Forum}

The 150 stakeholders at the National Prostate Cancer Forum formulated draft recommendations on the last day of the forum, which took place February 27 to March 2 . The draft recommendations will ultimately impact the supportive care, detection and diagnosis, and treatment and care of both survivors and men that will be diagnosed as having prostate cancer. The final recommendations, which will be released shortly, will focus on key areas of prostate cancer including research, communication and advocacy. A primary focus of the draft recommendations is a need for enhanced awareness on behalf of the public and health care profession regarding early detection practices. Information about the high incidence, morbidity and mortality of prostate cancer would also be targeted to the general public.

The stakeholders in the forum included prostate cancer survivors, health care professionals, researchers and governmental and organization representatives from across Canada. The draft recommendations will be approved and adopted through a process that includes all 150 stakeholders of the National Prostate Cancer
Forum. There will be a formal media announcement about the forum recommendations. In addition, a proceedings document will be developed to be published at a later date.

Highlights of the National Prostate Cancer Forum included:

- Dr. Ross Gray: Release of findings from the first-ever research report examining men's experiences, opinions and feelings about prostate cancer entitled, Canadian Men's Experiences with Prostate Cancer: A National Study.

- Debate on prostate specific antigen (PSA) screening

- Dr. Richard Cruess: Doctor and prostate cancer survivor

- Environmental issues and prostate cancer

- The genetic contribution to prostate cancer.

The National Prostate Cancer Forum was sponsored by the Canadian Cancer Society, the National Cancer Institute of Canada, Health Canada, ZENECA PHARMA Inc. and was co-sponsored by Abbott Laboratories and Schering Canada. 